\title{
Funcionamiento Familiar y Resiliencia en Escolares del nivel secundario Institución Educativa Estatal 20321 Santa Rosa, Huacho 2015
}

Family and resilience of operation in secondary school level state educational institution 20321 Santa Rosa, Huacho 2015

Hugo Teodoro Rojas Carranza', Olimpia Martha Camarena Lino', Martha Julia La Rosa Fabián², Aníbal Pantaleón

Sifuentes Damián ${ }^{3}$, Eudosia Adela Camarena Lino ${ }^{1}$, Henry Antonio Morales Gamarra ${ }^{1}$, Tania Zayda, Cuellar Camarena ${ }^{2}$

\section{RESUMEN}

Objetivo: Determinar la relación entre funcionamiento familiar y resiliencia en escolares del nivel secundaria de la Institución Educativa Estatal № 20321, Santa Rosa, Huacho, 2015. Método: Investigación básica, no experimental, correlacional transversal. La población de estudio fue de 109 adolescentes entre 14 a 17 años. Para la recolección de datos se utilizaron encuestas estructuradas, y el cuestionario FF-SIL para la variable Funcionamiento Familiar, el test RESI-M para la variable Resiliencia. En el análisis de datos se hicieron uso de tablas, gráficos y la prueba de significación de Rho de Spearman con el paquete estadístico SPSS v. 20 y hoja de cálculo Excel. Resultados: La mayoría $(51 \%)$ de estudiantes perciben el funcionamiento de su familia como moderadamente funcional, el (29\%) familias disfuncionales, (14\%) familias funcionales y solo el $(6 \%)$ familias severamente disfuncionales. Los resultados respecto a la Resiliencia el (65\%) de adolescentes tienen un nivel medio alto, el (32\%) un nivel medio bajo, y un (3\%) un nivel alto. Existe relación significativa entre el funcionamiento familiar y la resiliencia de las escolares del nivel secundaria de la Institución Educativa Estatal $\mathrm{N}^{\circ}$ 20321, Santa Rosa, Huacho, 2015; con $\mathrm{p}$ - valor $=0,000$ (prueba bilateral), con grado de relación $\mathrm{Rsp}=0,53$, lo que indica que es moderado y positivo. Conclusiones: Se evidencia que existe relación significativa entre el funcionamiento familiar y resiliencia en las escolares de nivel secundaria de la Institución Educativa Estatal № 20321, Santa Rosa, Huacho, 2015; es decir mientras el funcionamiento familiar sea bueno, mejor será el nivel de resiliencia de los escolares de la mencionada institución educativa.

Palabras clave: Funcionamiento familiar, resiliencia, escolares

\section{ABSTRACT}

Objective: To determine the relationship between family functioning and resilience in students of secondary school at 20321 Santa Rosa public school, Huacho, 2015. Method: Basic, nonexperimental, cross-correlational research. The study population consisted of 109 adolescents from 14 to 17 years. For the data collection, structured surveys were used, and the FF-SIL questionnaire for the family functioning variable, the RESI-M test for the Resilience variable. In the data analysis were used tables, graphs and Spearman's Rho significance test with the statistic package SPSS v. 20 and Excel spreadsheet. Results: The most $(51 \%)$ of students perceive their family functioning as moderately functional, (29\%) dysfunctional families, (14\%) functional families and only $(6 \%)$ severely dysfunctional families. The results respect to resilience $(65 \%)$ of adolescents have a medium high level, (32\%) a low average level, and (3\%) a high level. There is a significant relationship between family functioning and resilience in students of secondary school at 20321 Santa Rosa public school, Huacho, 2015; with $p$ - value $=0.000$ (bilateral test), with degree of relation $R s p=0.53$, indicating that it is moderate and positive. Conclusions: There is evidence that there is a significant relationship between family functioning and resilience in students of secondary school at 20321 Santa Rosa public school, Huacho, 2015; that is to say, while family functioning is good, better will be the level of resilience of students in the mentioned school.

Keywords: Family functioning, resilience, students. 


\section{INTRODUCCIÓN}

El estudio realizado por Barella, Mesa y Cobeña (2002) sobre conocimiento y actitudes sobre sexualidad de los adolescentes de nuestro entorno. Define la adolescencia como el periodo de la vida comprendido entre los 10 y 19 años.

Según Csikszentmihalyi, 1999; Grotberg, 1995; Rutter, 1985, et.al. Menezes de Lucena Carvalho, Fernández Calvo, Hernández Martín, Ramos Campos y Contador Castillo, (2006). La Resiliencia es la capacidad de sobreponerse a la adversidad, recuperarse, y salir fortalecido, con éxito y de desarrollar competencia social, académica y vocacional, pese a estar expuesto a un estrés psicosocial grave.

El Comité de Expertos de la OMS (1977) en Necesidades de Salud de los adolescentes. Ginebra Organización Mundial de la salud, sostiene que en la mayoría de las culturas el comienzo de la adolescencia se relaciona de una manera o de otra con la aparición de la pubertad, pero existen amplias diferencias, en cambio, en cuanto al criterio para determinar cuándo termina la adolescencia. Hay sin embargo un factor constante: aunque ya no es un niño, el adolescente no está considerado todavía por la sociedad como un verdadero adulto.

La familia funcional favorece a la resiliencia de las adolescentes escolares del nivel secundaria, pues al encontrase en una etapa de cambios y crisis, los hogares son el mejor soporte para su desarrollo. De ahí que, otro estudio realizado por autores como:

Nardone, Giannotti, Rocchi, (2012) en Modelos de Familia, sostiene que la familia es el sistema de relaciones fundamentalmente afectivas, presente en todas las culturas, en el que el ser humano permanece largo tiempo, y no un tiempo cualquiera de su vida, sino el formado de sus fases evolutivas cruciales (neonatal, infancia y adolescente).

Arenas, (2009). En su estudio Relación entre la funcionalidad familiar y la depresión en adolescentes. El objetivo fue: Determinar la relación existente entre la funcionalidad familiar y la depresión en los y las adolescentes atendidos en el Departamento de Psicología del Hospital Nacional Hipólito Unanue, Lima, Perú. Llegó a las conclusiones siguientes: La percepción de los niveles de funcionalidad familiar y su relación con la presencia o ausencia de depresión en adolescentes no presenta relación significativa en la muestra estudiada. Respecto a los niveles de funcionalidad que perciben los adolescentes de ambos géneros no se hallaron diferencias estadísticamente significativas, ya que la mayoría de los evaluados, independiente del género, perciben un nivel de rango medio en la funcionalidad familiar.

Carballo y Lescano (2012). Estudio sobre Funcionamiento familiar y rendimiento académico en estudiantes de secundaria de la institución educativa $\mathrm{N}^{\circ} 0292$ - Tabalosos. Marzo 2011 - abril 2012. El objetivo de la investigación fue: Identificar la relación existente entre el funcionamiento familiar y el nivel de rendimiento académico de los estudiantes del 5to grado del nivel secundario de la Institución Educativa No 0292 del Distrito de Tabalosos, durante el periodo marzo 2011 y abril 2012. Obtuvieron las siguientes conclusiones según el tipo de familia el riesgo de vulnerabilidad disfuncional se presentó $25 \%$ (07 familias). Según el número de miembros e hijos en el hogar el 48\% (12 familias) tienen mayor vulnerabilidad hacia la disfuncionalidad familiar y $57,1 \%$ son menos vulnerables. La etapa del ciclo vital de la familia según edad del hijo/a mayor indican que el 17,9\% (05 alumnos) tienen mayor riesgo de vulnerabilidad funcional en la familia y necesitan mayor flexibilidad y capacidad de adaptación a la crisis. Según jerarquía y límites en el hogar el 89,2\% (25 estudiantes) tienen una clara percepción del rol del padre o madre y de autoridad dentro de la familia y sólo el 3,6\% tienen tendencias al caos dentro de la organización familiar. En cohesión familiar el $50 \%$ (14 estudiantes) muestran mayor apego emocional, mayor sentimiento de unión y una más explícita expresión de afecto entre los miembros de la familia y solo 7,1\% (02 estudiantes) tienen propensión a dificultades emocionales y del comportamiento.

Graza, (2013) en su estudio Relación entre funcionalidad familiar y nivel de violencia escolar en los adolescentes de la Institución Educativa Francisco Bolognesi Cervantes $N^{\circ}$ 2053 Independencia 2012. Lima, cuyo objetivo fue: Determinar la relación entre funcionalidad familiar y nivel de violencia escolar en los adolescentes de la Institución Educativa Francisco Bolognesi Cervantes $N^{\circ} 2053$. El tipo de investigación fue cuantitativo, de nivel aplicativo, La población de estudio estuvo 
conformada por todos los adolescentes de 14 19 años de ambos sexos, pertenecientes al $4^{\circ} \mathrm{y}$ $5^{\circ}$ año de secundaria, mediante el muestreo probabilístico aleatorio simple Conclusiones: La mayoría de los adolescentes del 4to. Y 5to. Año de secundaria de la I.E. Francisco Bolognesi Cervantes (53\%) provienen de una familia disfuncional, es decir, de aquellas que presentan situaciones de convivencia llenas de estrés; presentando dificultades como la disminución del autoestima, bajo rendimiento académico, estrés, depresión, desmotivación y conductas sumisas, lo que les lleva a ser vulnerables ante abusos por parte de sus compañeros. La mayoría de los adolescentes del 4to y 5to año de secundaria de la I.E. Francisco Bolognesi Cervantes presentan niveles altos de violencia escolar en un $49 \%$, con una predominancia de la violencia psicológica del $84 \%$, que se traduce en amenazas, insultos, poner apodos, destacando éste último. Existe relación estadísticamente significativa entre la funcionalidad familiar y el nivel de violencia escolar de los adolescentes del 4to y 5to años de secundaria de la I.E. Francisco Bolognesi Cervantes específicamente con familias disfuncionales, pero también se concluye que las familias funcionales de los adolescentes estudiados son generadoras de violencia escolar, existiendo en este grupo otros factores de importancia como es el entorno social en el que se desenvuelven. Con respecto a la hipótesis: "Existe relación significativa entre funcionalidad familiar y nivel de violencia escolar en los adolescentes de la I.E Francisco Bolognesi Cervantes № 2053", esta se acepta, por lo tanto, los resultados obtenidos en la investigación ratifican la relación que existe entre la funcionalidad familiar y el nivel de violencia escolar; es decir, una disfuncionalidad familiar, genera que se presente la violencia en los adolescentes.

Existe violencia escolar en la institución educativa estudiada, la misma que responde a su característica singular de tener capacidad para multiplicarse y para expandir sus dinámicas y sus consecuencias. La investigación marca el alcance de la violencia escolar en porcentajes superiores al $49 \%$ para el nivel alto, $27 \%$ para el nivel medio y en $24 \%$ para el nivel bajo.

Flores, (2008) en Resiliencia y proyecto de vida en estudiantes del tercer año de secundaria de la UGEL 03, Lima, Perú. El objetivo fundamental de esta investigación fue determinar si existe relación entre los niveles de resiliencia y el grado de definición del proyecto de vida en estudiantes del tercer año de secundaria de colegios nacionales y particulares de la UGEL 03. La técnica de muestreo ha sido no probabilística, se seleccionó una muestra de estudiantes de ambos sexos (Varones $=200$; Mujeres $=200$ ). La investigación fue de tipo descriptivo correlacional. Los resultados permiten llegar a la conclusión de que existe relación altamente significativa ( $p$ relación altamente significativa $(p<0,0001)$ entre el grado de resiliencia y el grado de definición del proyecto de vida, tanto en la muestra de estudiantes varones como en la de mujeres.

Zegarra A. (2013) en Factores asociados con la capacidad de resiliencia, en adolescentes de la I. E. "Luis Alberto Sanchez" Viñani, Distrito Gregorio Albarracín Tacna 2012. La investigación tuvo un diseño descriptivo con enfoque cuantitativo de tipo transversal correlacional; con el objetivo de "Determinar los factores asociados con la capacidad de resiliencia, en los adolescentes de la I. E. "Luis Alberto Sánchez" Viñani, Distrito Gregorio Albarracín Tacna 2012". El universo está conformada por todos los estudiantes del nivel secundario un total de 686 alumnos, La muestra fue de 181 alumnos obtenidos estadísticamente mediante la fórmula para poblaciones finitas, el instrumento de medición que se utilizó, es el cuestionario estructurado auto aplicativo. Una vez obtenida la información se ordenó, codificó y procesó utilizando el software estadístico informático Statistical Package for the Social Sciences (SPSS). Las conclusiones fueron: La mayoría de adolescentes de la I. E. Luis Alberto Sánchez presentaron una capacidad de resiliencia media. El factor protector predominante es la familia $(59,7 \%)$ y la Institución Educativa $(59,1 \%)$ que la comunidad en el desarrollo de la capacidad de resiliencia del adolescente de I. E. Luis Alberto Sánchez. Se determinó que los factores asociados con la capacidad de resiliencia del adolescente $(p<0,05)$, son factores protectores y factores de riesgo y tiene un nivel alto de significancia en el desarrollo de la capacidad de resiliencia $(p<0,05)$.

Finalmente, habiendo tomado como referencias otros estudios similares respecto a 
las dos variables en estudio, la presente investigación se diferencia de cada una de las anteriores, por las siguientes razones:

Es una población únicamente femenina, púber y adolescente del nivel secundario, quienes presentan problemas familiares, donde medimos los niveles de funcionalidad de las familias desde la percepción de las estudiantes y los niveles de resiliencia frente a los problemas o dificultades que presentan. Que, a la vez, permitirán presentar propuestas preventivas de intervención multidisciplinaria. Que redundará en beneficio de la institución educativa y de las escolares que se beneficiaran del aporte profesional.

El objetivo fue determinar la relación entre funcionamiento familiar y resiliencia en escolares del nivel secundaria de la Institución Educativa Estatal $N^{\circ}$ 20321, Santa Rosa, Huacho, 2015.

\section{MATERIAL Y METODOS}

El tipo de investigación que se desarrollo fue básica, descriptiva y correlacional, con un enfoque cuantitativo de corte transversal. Para la recolección de información se utilizó la técnica de encuesta, para la medición del funcionamiento familiar el cuestionario FF SIL diseñado y validado por Ortega De la Cuesta y Días en el año 1999. Para la medición de la variable resiliencia se utilizó la Escala de Resiliencia, Wolin (adaptación Instituto Vivir).

El procesamiento, organización presentación, análisis e interpretación de los datos se hicieron en Tablas, Figuras estadísticas, porcentajes y la prueba de significación de Rho de Spearman, utilizando el paquete estadístico SPSS v. 20 y la hoja de cálculo EXCEL.

\section{Población y Muestra}

Participaron en el estudio 109 alumnas, comprendidos entre las edades de 14 - 18 años del cuarto y quinto año de secundaria Institución Educativa $N^{\circ}$ 20321, Santa Rosa, Huacho, 2015. Todas las participantes fueron mujeres por las características ya establecidas en la institución educativa.

\section{RESULTADOS}

Tabla 1. Edad de los escolares

\begin{tabular}{ccr}
\hline Edad & Frecuencia & $\%$ \\
\hline 14 & 3 & 3 \\
15 & 41 & 37 \\
16 & 52 & 48 \\
17 & 11 & 10 \\
18 & 2 & 2 \\
Total & 109 & 100 \\
\hline
\end{tabular}

En la Tabla 1 se observa que las edades de las escolares que participaron en la investigación, fluctúan entre los 14 a 18 años, donde la mayoría se encuentra entre los $16(48 \%)$ y 15 (37\%) años, un tercer grupo tiene $17(10 \%)$, un cuarto grupo de escolares tiene 14 años (3\%) y también existe estudiantes en el nivel secundaria con 18 años $(2 \%)$.

Tabla 2. Percepción del funcionamiento Familiar de las escolares

\begin{tabular}{lccr}
\hline Funcionamiento Familiar & Rango & Frecuencia & $\%$ \\
\hline Familia Funcional & $57-70$ & 15 & 14 \\
Familia Moderadamente Funcional & $43-56$ & 56 & 51 \\
Familia Disfuncional & $28-42$ & 32 & 29 \\
Familia Severamente Disfuncional & $14-27$ & 6 & 6 \\
\multicolumn{1}{c}{ Total } & & 109 & 100
\end{tabular}

En la Tabla 2 se observa la percepción de las escolares respecto al funcionamiento de su familia, la mayoría (51\%) percibe a su familia en el rango moderadamente funcional, (29\%) percibe que su familia como disfuncional, el (14\%) percibe a su familia como funcional, y el $(6 \%)$ de escolares percibe a su familia severamente disfuncional.

Tabla 3. Nivel de Resiliencia de las escolares

\begin{tabular}{lrcr}
\hline Niveles de Resilencia & Rango & Frecuencia & $\%$ \\
\hline Nivel alto & De $1-42$ & 0 & 0 \\
Nivel medio alto & $43-84$ & 71 & 65 \\
Nivel medio bajo & $84-126$ & 35 & 32 \\
Nivel bajo & $127-168$ & 3 & 3 \\
\multicolumn{1}{c}{ Total } & & 109 & 100 \\
\hline
\end{tabular}

Según la Tabla 3 se ha obtenido como resultado que la resiliencia en la mayoría de las escolares $(65 \%)$ se encuentra en el nivel medio alto, el (32\%) está en nivel medio bajo, el $(3 \%)$ de las escolares tienen un nivel bajo y $(0 \%)$ de escolares nivel alto de resiliencia. 


\section{Hipótesis General}

$\mathrm{H}_{0}$ : $\quad$ No existe relación entre funcionamiento familiar y la resiliencia de las escolares del nivel secundaria de la Institución Educativa Estatal №20321, Santa Rosa, Huacho, 2015.

$\mathrm{H}_{1}$ : $\quad$ Existe relación entre funcionamiento familiar y la resiliencia de las escolares del nivel secundaria de la Institución Educativa Estatal N²0321, Santa Rosa, Huacho, 2015.

\section{Resultados de la prueba}

Los resultados de la prueba, $p$ - valor $=0,000$ (prueba bilateral) es menor a $\alpha=0,05$; esto implica que hay evidencias suficientes para afirmar que existe relación significativa entre el funcionamiento familiar y la resiliencia de las escolares del nivel secundaria de la Institución Educativa Estatal No 20321, Santa Rosa, Huacho, 2015, siendo el grado de relación moderado y positivo, cuyo valor es $R_{s p}=0,53$. Es decir, mientras el funcionamiento familiar sea bueno, mejor será el nivel de resiliencia de las escolares.

\section{Hipótesis Específicas Primera hipótesis estadística}

$\mathrm{H}_{0}$ : $\quad$ No existe relación entre funcionamiento familiar y la resiliencia de las escolares púberes (11 a 14 años) del nivel secundaria de la Institución Educativa Estatal $N^{\circ} 20321$, Santa Rosa, Huacho, 2015.

$\mathrm{H}_{1}$ : Existe relación entre funcionamiento familiar y la resiliencia de las escolares púberes (11 a 14 años) del nivel secundaria de la Institución Educativa Estatal No 20321, Santa Rosa, Huacho, 2015.

\section{Resultados de la prueba}

Los resultados de la prueba, $\mathrm{p}$ - valor $=$ 0,078 (bilateral) es menor a $\alpha=0,05$; esto implica que hay evidencias suficientes para afirmar que existe relación significativa entre funcionamiento familiar y la resiliencia de las escolares púberes (11 a 14 años) del nivel secundaria de la Institución Educativa Estatal $N^{\circ} 20321$, Santa Rosa, Huacho, 2015, siendo el grado de relación moderado y positivo, cuyo valor es $R_{s p}=0,46$. Es decir, mientras el funcionamiento familiar sea bueno, mejor será el nivel de resiliencia de los escolares púberes.

\section{Segunda hipótesis estadística}

$\mathrm{H}_{0}$ : $\quad$ No existe relación entre funcionamiento familiar y resiliencia de las escolares adolescentes (15 a 17 años) del nivel secundaria de la Institución Educativa Estatal N²0321, Santa Rosa, Huacho, 2015.

$\mathrm{H}_{1}$ : Existe relación entre funcionamiento familiar y resiliencia de las escolares adolescentes (15 a 17 años) del nivel secundaria de la Institución Educativa Estatal N²0321, Santa Rosa, Huacho, 2015.

\section{Resultados de la prueba}

Los resultados de la prueba, $\mathrm{p}$ - valor $=$ 0,02 (bilateral) es menor a $\alpha=0,05$; esto implica que hay evidencias suficientes para afirmar que existe relación significativa entre funcionamiento familiar y resiliencia en las escolares adolescentes (15 a 17 años) del nivel secundaria de la Institución Educativa Estatal $N^{\circ} 20321$, Santa Rosa, Huacho, 2015, siendo el grado de relación moderado y positivo, cuyo valor es Rsp $=0,39$. Es decir, mientras el funcionamiento familiar sea bueno, mejor será el nivel de resiliencia de los escolares adolescentes.

\section{DISCUSION}

Según los resultados obtenidos en la presente investigación y luego de la contrastación de las hipótesis, se puede afirmar que existe relación significativa entre funcionamiento familiar $y$ resiliencia en escolares de nivel secundaria, cuyo grado de relación es positivo y medio de la Institución Educativa Estatal 20321 Santa Rosa, Huacho 2015. Esta investigación coincide con los de Pérez, Illacutipa y Guedez (2015), quienes concluyen que existe relación significativa entre las dimensiones del funcionamiento familiar y la Resiliencia en estudiantes con facultades sobresalientes del tercer año de secundaria con grado de relación positiva y media de una institución educativa pública con altas exigencias académicas durante el año 2013. Por tanto, mientras mejor sea el nivel de funcionamiento familiar a un mejor será el nivel de resiliencia.

Por otro lado, en nuestra investigación se obtuvieron hallazgos referentes a resiliencia de 
los escolares en cuales la mayoría de los escolares presentaron un nivel de resiliencia positivo medio alto. Coincidentemente Zegarra A. (2013). En su estudio Factores asociados con la capacidad de resiliencia, en adolescentes de la I. E. "Luis Alberto Sánchez" Viñani, Distrito Gregorio Albarracín Tacna 2012.- concluye que la mayoría de adolescentes de la institución educativa presentan una capacidad de resiliencia media. El factor protector predominante es la familia y la Institución Educativa, antes que la comunidad en el desarrollo de la capacidad de resiliencia del adolescente.

Finalmente, habiendo tomando como referencia otros estudios respecto a las dos variables en estudio, se sugiere realizar actividades preventivas a través de talleres con equipos multidisciplinarios con participación de los padres de familia y escolares, que redundara en la mejora del nivel de funcionamiento familiar y resiliencia.

\section{Agradecimientos}

Alas escolares adolescentes del cuarto y quinto años de secundaria de la Institución Educativa Estatal N 20321 Santa Rosa, 2015, a sus docentes y autoridades, por haber contribuido desinteresadamente, en la realización de la presente investigación.

\section{REFERENCIAS BIBLIOGRÁFICAS}

Arenas, S. (2009). Relación entre la funcionalidad familiar y la depresión en adolescentes. Tesis para optar título de Licenciada en psicología. Lima: Universidad Nacional Mayor San Marcos.

Barella Balboa, J. L., Mesa Gallardo, I. \& Cobeña Manzorro, M. (2002). Conocimientos y actitudes sobre sexualidad de los adolescentes de nuestro entorno. Medicina de Familia, 4(3), 37-42.

Carballo, G. \& Lescano, E. (2012). Funcionamiento familiar y rendimiento académico en estudiantes de secundaria de la institución educativa $N^{\circ} 0292$ - Tabalosos. Marzo 2011 - abril 2012. Tesis para optar el título profesional de Licenciada en Enfermería. Universidad nacional de San Martin, Tarapoto.

http://www.unsm.edu.pe/spunsm/archivos _proyectox/archivo_98_Binder1.pdf
OMS (1977). Necesidades de salud de los adolescentes: informe de un Comité de Expertos de la OMS, (Ginebra, 1976, Septiembre, 13).

Flores, M. (2008). Resiliencia y proyecto de vida en estudiantes del tercer año de secundaria de la UGEL 03. Tesis para optar el grado de Magister en Psicología, Lima: Universidad Nacional Mayor de San Marcos.

Pérez, T. J. G., Illacutipa, Z. K. Y. \& Guedez, K. P. (2015). Funcionamiento familiar y resiliencia en estudiantes con facultades sobresalientes del tercer año de secundaria de una institución educativa pública con altas exigencias académicas, durante el período 2013. Revista Científica de Ciencias de la Salud, 7(2), 29-34.

Graza, S. (2013). Relación entre funcionalidad Familiar y nivel de violencia escolar en los adolescentes de la institución educativa Francisco Bolognesi cervantes No 2053 Independencia 2012. Tesis para optar el título de Licenciado de enfermería, Lima: Universidad Nacional Mayor de San Marcos.

Menezes de Lucena Carvalho, V. A., Fernández Calvo, B., Hernández Martín, L., Ramos Campos, F., \& Contador Castillo, I. (2006). Resilencia y el modelo Burnoutengagement en cuidadores formales de ancianos. Psicothema (Oviedo), 791-796.

Nardone, G., Giannotti, E. \& Rocchi, R. (2012). Modelos de Familia. España: Herder.

Zegarra, A. (2013). Factores asociados con la capacidad de resiliencia, en adolescentes de la I. E. "Luis Alberto Sánchez " Viñani, distrito Gregorio Albarracín Tacna 2012. Tesis para optar el título de Licenciado en enfermería. Tacna: Universidad Nacional Jorge Basadre Grohmann.

Correo electrónico: hrojas@unjfsc.edu.pe

Revisión de pares:

Recibido: 28-10-2016

Aceptado: 19-12-2016 